UDC 336.743

DOI: $10.24045 /$ et.2017.4.3

\title{
BITCOIN IS THE CURRENCY OF THE FUTURE
}

Yu. A. Petrova

A. D. Roldugina

O. A. Kartseva
Candidate of Philosophical Sciences, assistant professor, e-mail: Julia-pp@yandex.ru, undergraduate students, e-mail: Julia-pp@yandex.ru e-mail: Julia-pp@yandex.ru

Rostov State University of Economics, Rostov-on-Don, Russia

\begin{abstract}
In the modern era, the digital currency or "crypto-currency" is developed rapidly. The most famous and large-scale digital payment system is bitcoin, the operation of which is based on modern blockchain technology. This crypto currency was invented by Satoshi Nakamoto in 2008. Nowdays, this system operates in many countries in the world, and the number of such countries is growing every year.
\end{abstract}

Key words: bitcoin; blockchain; currency; payment; transactions; miners.

Bitcoin is a worldwide anonymous crypto currency and digital payment system [5]. This currency is the first digital decentralized currency, there is no central repository and administrators. Thus, the payment between two people is irreversible and without intermediaries.

Bitcoins is a convenient currency for people as means for their use, for example it has reliable protection from control of banks and states, and also the crypto currency has anonymity of transactions, since transactions. To use bitcoins, you need only an access to the Internet, another advantage is no commission for all transactions, or it is very small. But the main property of bitcoins is the high confidence of their users, since the payment system by bitcoins has huge computing resources all over the world, and also has a reliable mathematical base that is not subject to various attacks.

In October 2008, the Japanese Satoshi Nakamoto published his invention in the research paper entitled "Bitcoin: A Peer-toPeer electronic Cash System" [2]. In the first days of the invention of this payment system, Satoshi Nakamoto produced one million bitcoins according to estimates [4]. Hal Finney is one of the first contributors to the new crypto-currency system. After downloading bitcoin software on the day of its release,
Satoshi Nakamoto decided to surprise the first supporters and transferred 10 bitcoins to him. The first exchange of bitcoins occurred in September 2009. After the presentation of ac new Nakamoto crypto currency, he transferred his creation to Gavin Andresen, who soon became the leading bitcoin developer in Bitcoin-Fund [1]. In August 2017, bitcoin was divided into two types: classical bitcoin (BTC) and bitcoin-cash (BCH) [4].

At the heart of bitcoin lies the chain of transactions (blockchain), built according to certain rules, which contains information about all transfers of electronic currency bitcoins. Block as a distributed database consistently consists of blocks - a list of ordered data about the financial transactions committed by participants. All copies of the database are stored in bitcoin wallets, which users of bitcoins create for themselves. An access to the online wallet is possible from a computer, phone or tablet. With the help of online wallet, each of the transactions are made, confirmed and fixed in a certain block. Each user receives his personal address, passing the registration, which you need to specify during the transaction bitcoin. This address ensures the complete confidentiality of the person. The purse includes: public address, balance and private key. The public address and private key are the login and password, so 
that you can transfer bitcoins and must know your login to send the bitcoins, but you only need to know the private key and not to tell this code to anyone else.

However, the main role in the functioning of the system is played by the "miners" people who process the transactions and form blocks of them. The creation of the block is the construction of a set of hash codes due to asymmetric algorithms. Hashes contain a reference to the previous block with its transactions, as well as those transactions that were committed, but are not currently recorded. For the creation of one block, a bonus is accrued, a kind of salary, so the creation of blocks is usually called "mining of bitcoins", that is, "mining" of electronic currency. But this reward for the found signature of the block decreases every year.

The mining process is a process that can be compared with the mining of minerals and requires the solution of a number of complex mathematical problems, so the miners need to use powerful electronic computing resources, which computers equipped by special microcircuits and programs.

The blockchain is a brilliant technology of the future, where the authenticity of money transfer transactions is checked only by its users, without regulators and intermediaries which can serve as an identifier for ownership of land and diamonds, allows maintaining copyright and developing anonymous voting system. Despite the fact that the bitcoin rate is minimal, there are tendencies for growth. It has grown approximately five times. Prior to widespread use of bitcoin in world trade is still far away, but in a number of countries it has already been accepted as a legal tender. Blockchain remains at the center of attention of large banking networks, technology corporations, governments and venture investors. In November 2016, the total investment in the projects of blockchain and bitcoin exceeded one billion dollars.

To date, there are no laws that regulate the turnover of the crypto currency inside the state, but there is also no ban on it. The question of the legality of the use of bitcoins by people in different countries remains controversial, since we can not tax settlement oper- ations in the crypt, and, because of the $100 \%$ anonymity of transactions, so you can make various purchases of goods without identity their personality.

The popularity of this crypto currency is growing every day. The most active bitcoins are used in such countries as the USA, South Korea, the Netherlands, Finland, etc.

The largest number of users of the crypto-currency economy and bitcoins is in the US. There is a huge number of Bitcoin ATMs in the country. In addition, the United States is a global financial superpower, and various countries around the world are turning there for guidance on the legal status of the crypto currency and regulatory clarity. Thus, the US will serve as a platform for crypto-regulation in the coming years, while others will build on their experience.

Like the US, South Korea develops rapidly a new crypto currency as a means of exchange and investment. The most powerful bitcoins are used in a dynamic technical industry, which includes supercorporations, such as LG and Samsung. Also, South Korea is a regular guest at conferences, the topics of which are the develop of bitcoins and the improvement of this payment system. In Korea there is a secure Corbit service, thanks to which it is possible to accept, buy and use bitcoins. In addition to Corbit, a lot of other services appears due to the fact that there are no laws in the country that would regulate the crypto-exchange payment system. People can buy bitcoins in certain stores in South Korea.

Friendly to bitcoin country is Netherlands. Here is the unique city of Arnhem, where you can pay all services for by bitcoins, including payments for gas, housing, bicycles and even dental services. In the Netherlands there is a large number of Bitcoin ATMs and in the center of Amsterdam there is an embassy bitkoin. The Netherlands is a regular participant of conferences with the main topic about "bitcoin".

International trade plays an important role for the future of the bitcoins [6]. Every time when we pay by a credit card in the shops, we pay a commission. That is why more and more companies such as Wikipe- 
dia, WordPress Overstock, use a new crypto currency at present time.

Micropayments are the trend of the last year is certainly. Now access to the Internet, people can pay by bitcoins for music, computer games and so on, though earlier they could only buy a subscription for a certain period.

It can be said that in the modern era of crypto currency, bitcoin can serve as a basis for creating an earlier decentralized economy, which will be controlled only by participants in the market system.

\section{Bibliography}

1. Bosker B. Gavin A., Bitcoin Architect: Meet The Man Bringing You Bitcoin (And Getting Paid In It), HuffPost American news and opinion, 2016. [Retrieved from: https://www.huffingtonpost.com/2013/04/16/gavi n-andresen-bitcoin_n_3093316.html]
2. Brito J. \& Castillo A., Bitcoin: A Primer for Policymakers. Mercatus Center George Mason University, 2013.

3. Smith J. The Bitcoin Cash Hard Fork Will Show Us Which Coin Is Best, 2017. Fortune is a multinational business magazine, published and owned by Time Inc. and headquartered in New York City. [Retrieved from: http://fortune.com/2017/08/11/bitcoin-cash-hardfork-price-date-why/]

4. McMillan R., Who Owns the World's Biggest Bitcoin Wallet? 2016. [Retrieved from: https://www.wired.com/2013/12/fbi_wallet/]

5. Satoshi N. Bitcoin: A Peer-to-Peer Electronic Cash System, 2014. [Retrieved from: www.bitcoin.org]

6. Retrieved from: http://www.forbes.ru/tekhnologii/internet-isvyaz/317341-sdvig-paradigmy-kak-bitkoinizmenit-finansovuyu-sistemu

(C) Petrova Yu.A., Roldugina A. D. Kartseva O. A., 2017. 